\title{
METALLICITY OF TWO STARS IN BAADE'S WINDOW*
}

\author{
S. CASTRO ${ }^{1}$, B. BARBUY ${ }^{1}$, T. RICHTLER ${ }^{2}$ \\ 1 IAG-USP, São Paulo, Brazil; 2 Bonn Univ., Germany
}

\begin{abstract}
Low resolution spectra of two stars of the Baade Window were obtained with the $1.5 \mathrm{~m}$ telescope at ESO. Their temperatures and gravities are obtained by using their location in colour magnitude diagrams of bulge clusters, and through synthetic spectra fitting, their metallicities are derived.
\end{abstract}

\section{Introdution}

Whitford \& Rich (1983) and Rich (1988, 1992) described the stellar populations of the galactic bulge, in particular that of the Baade Window (BW). The bulge contains a distinct stellar population relative to other components of the Galaxy, including RR Lyrae, M giants, OH/IR stars and Miras. The metallicity of bulge stars in the BW range from $1 / 10$ to 10 times solar, according to Rich.

Further studies on the metallicity of BW's stars are of great interest, and this poster addresses the study of two BW stars for which we have low resolution spectra.

\section{Observations and Stellar Parameters}

The observations were obtained by T. Richtler at the $1.5 \mathrm{~m}$ telescope of the European Southern Observatory - ESO, at La Silla (Chile). The Image Dissector Scanner IDS was used as detector. Mid-resolution spectra of $\Delta \lambda \approx 5 \AA$ were obtained in the wavelength range $\lambda \lambda 400-620 \mathrm{~nm}$ for the stars 2 and 9 of the Baade Window's field near NGC 6528 according to the identifications by van den Bergh (1971). The BV colours from van den Bergh (1971) are used to derive the temperature, using the method presented in Barbuy et al. (1992). The reddening $E(B-V)=$ 0.58 indicated by van den Bergh \& Younger (1979) was adopted, pointing out that this is a mean between the values $E(B-V)=0.55$ found for the direction of the globular cluster NGC 6528 by Ortolani, Bica \& Barbuy (1992, OBB92), and the mean values $\mathrm{E}(\mathrm{V}-\mathrm{I})=0.70$ given by Walker \& Mack (1986) and adopted by Sadler (1992) for the Baade Window.

Below are shown their colours and derived parameters. For star 9, it could be ascent $R G B$ or RGB tip which in (B-V) mixes either the hotter stars (see OBB92).

\begin{tabular}{lcccc}
\hline star & $\mathrm{V}$ & $(\mathrm{B}-\mathrm{V})$ & $\mathrm{T}_{\mathrm{ef}}(\mathrm{K})$ & $\log \mathrm{g}$ \\
\hline 2 & 15.66 & 2.05 & 4000 & 0.71 \\
9 & 16.03 & 1.99 & 4100,3500 & 0.95
\end{tabular}

* Observations collected at the European Southern Observatory, La Silla, Chile 


\section{Derivation of Metallicity}

The MgI triplet plus continua at 489.7-495.8 and 530.3-536.6 nm are used here: we computed synthetic spectra for the region $\lambda \lambda 490-540 \mathrm{~nm}$, which are compared to the observed spectra.

Model atmospheres are interpolated in the grid by Gustafsson et al. (1975), using the same assumptions used in the calculations of the models.

In figure 1 are shown the synthetic spectrum, computed with $[\mathrm{M} / \mathrm{H}]=0.0$, fitted to star 2 in the range $\lambda \lambda 505-516 \mathrm{~nm}$. The star 9 proved to be a cool M giant of metallicity also approximately solar, where TiO bands dominate.

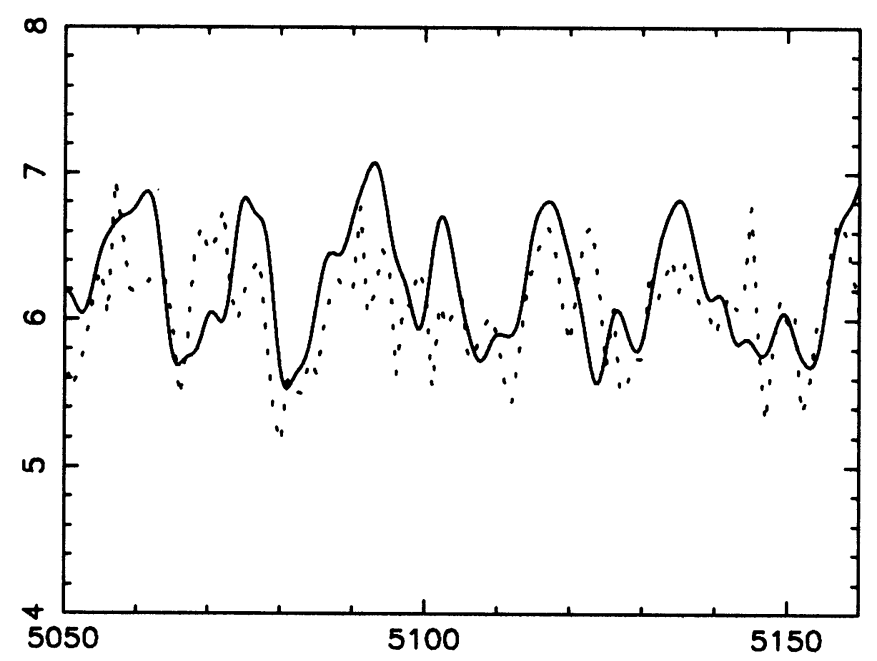

Fig. 1 - Synthetic spectrum (-) of star 2, computed with $[\mathrm{M} / \mathbf{H}]=0.0$

\section{References}

Barbuy, B., Castro, S., Ortolani, S., Bica, E.: 1992, A\&A 259, 607

Bica, E., Barbuy, B., Ortolani, S.: 1991, ApJ Lett. 382, L15

Gustafsson, B., Bell, R.A., Eriksson, K., Nordlund, A.:1975, A\&A 42, 407

Ortolani, S., Bica, E., Barbuy, B.: 1992, A\&AS 92, 441

Schmidt-Kaler, A.: 1982, in Landolt-Börnstein, group VI, vol. 2, subvol. b (Stars and star clusters)

Rich, M.: 1988, AJ, 95, 828

Rich, M.: 1992, in The Stellar Populations of Galaxies, IAU Symp. 149, eds. B. Barbuy, A. Renzini, Kluwer Academic Publishers, p. 29

Sadler, E.: 1992, in The Stellar Populations of Galaxies, IAU Symp. 149, eds. B.

Barbuy, A. Renzini, Kluwer Academic Publishers, p. 41

van den Bergh, S.: 1971, AJ 76, 1082

Walker, A.R., Mack, P.: 1986, MNRAS, 220, 69

Whitford, A., Rich, M.: 1988, ApJ 274, 723 